\title{
A survey of pregnant smokers' interest in different types of smoking cessation support
}

\author{
Michael Ussher*, Robert West, Nicola Hibbs \\ Department of Psychology, Hunter Wing, St. George's Hospital Medical School, \\ University of London, Cranmer Terrace, London SW17 ORE, UK
}

Received 22 October 2002; received in revised form 8 March 2003; accepted 9 June 2003

\begin{abstract}
The aim of this study was to survey pregnant smokers' interest in different types of smoking cessation support. Interest in cessation support was assessed via telephone interviews with women identified as smokers at their first antenatal visit. Of 206 pregnant smokers interviewed, 87\% (179/206) reported wanting to stop smoking, of whom 69\% (124/179) expressed an interest in receiving help with stopping. Interest was highest for behavioural support $(82 \%, 102 / 124)$ and self-help materials $(77 \%, 95 / 124)$. There was a significant preference for individual versus group appointments, for 'buddying' among those not in professional/managerial occupations versus those in professional/managerial occupations, and for behavioural support among non-Caucasians versus Caucasians. These findings highlight the high level of interest expressed in support with stopping smoking among pregnant smokers. Smoking cessation services may benefit through offering a range of interventions for pregnant smokers, through considering ethnic and occupational status, and through routinely offering individual appointments.
\end{abstract}

(C) 2003 Elsevier Ireland Ltd. All rights reserved.

Keywords: Behavioural support; Interests; Pregnancy; Smoking cessation; Survey

\section{Introduction}

Smoking during pregnancy is harmful for both the fetus and the mother, as well as for the developing child [1] and the resulting costs to health care organisations are substantial [2]. In industrialized countries between one in five and one in three pregnant women report smoking [3] and in the UK an estimated $27 \%$ of women continue to smoke throughout their pregnancy [4].

Stopping smoking is one of the most important actions a pregnant smoker can undertake to improve the outcome of her pregnancy [5]. Up to a quarter of women who smoke before their pregnancy are likely to stop smoking before their first antenatal visit without professional help [3]. A further $10 \%$ are likely to stop without support following the first antenatal visit [3] and the large majority of women who continue to smoke during their pregnancy are likely to want to stop $[4,6]$. Brief opportunistic advice by a physician can increase cessation rates in pregnancy by $1-2 \%$ [7]. More intensive counseling-based behavioural support has been shown to increase these rates to around 7\% [7].

\footnotetext{
* Corresponding author. Tel.: +44-20-8725-5605; fax: +44-20-8767-2741.

E-mail address: mussher@sghms.ac.uk (M. Ussher).
}

Despite the efficacy of behavioural support for smoking cessation during pregnancy a low level of uptake of this support has been cited as a major barrier to the implementation of such programmes [8-11]. A recent survey in the UK found that only around 5\% of pregnant smokers make use of the behavioural support for smoking cessation which is available free in the National Health Service [9].

Self-help materials may help about $4 \%$ of pregnant smokers to stop [3], although the level of interest in this type of support is not known. Whilst, telephone help-lines have been reported to be effective for increasing rates of smoking cessation among smokers in general [12], and the UK has a telephone help-line for pregnant smokers. Yet the level of interest in telephone help-lines among pregnant smokers is unknown.

Adding quite intensive supervised exercise to a behavioural treatment has been shown to increase smoking abstinence rates among women smokers [13]. No reports could be identified which have examined exercise interventions for pregnant smokers or which have assessed the likely interest in such interventions among these women.

There is some evidence for social support interventions ('buddy systems') increasing smoking abstinence rates in the general population [14] and lack of social support has been cited as the principle reason for poor attendance at 
smoking cessation sessions during pregnancy [11]. As yet there is very limited evidence for the benefits of the buddy system for pregnant smokers [15] and the level of interest in this approach has not been assessed for these women.

Poor uptake of smoking cessation support during pregnancy is regarded as a serious problem $[9,10,16]$. Whilst, it has been acknowledged that for smoking cessation interventions to be effective they must be acceptable to pregnant smokers [17]. An understanding of pregnant womens' levels of interest in, and preferences for, different smoking cessation interventions is likely to aid in developing interventions which are acceptable for these women $[18,19]$. Furthermore, it has been demonstrated that different smoking cessation strategies may be appropriate for different sub-populations of pregnant smokers [20]; for example, it has been shown that pregnant smokers from lower socio-economic groups are likely to respond better to more intensive behavioural interventions [20]. Previous work has examined interests in, and preferences for, different smoking cessation support among smokers in general [21-23]. No previous study could be identified examining pregnant womens' interests in different types of support with stopping smoking. This paper reports a survey to assess interests in, and preferences for, smoking cessation support among pregnant smokers.

\section{Methods}

\subsection{Sample and procedures}

Pregnant smokers were identified using the Patient Administration System (PAS) of a large district general hospital in Tooting, south west London. The survey took place across an 8-month period (June 2000-February 2001) prior to the establishment of a national network of pregnancy specific smoking cessation services in the UK. Approval was obtained from the local ethics committee and participants gave consent via the telephone.

Previously, brief telephone interviews have been used to assess preferences for smoking cessation support among smokers in general $[22,23]$. In the present study all those pregnant women identified as smokers at their first antenatal booking visit were telephoned within 1 week of their booking visit and were invited to take part in the survey. The women were interviewed via telephone on a single occasion for around $10 \mathrm{~min}$. The interview took place during the initial telephone call or within $48 \mathrm{~h}$ of this call. Women who could not be reached following four telephone calls were recorded as non-contactable. Those declining to take part in the survey were also recorded.

\subsection{Questionnaire items}

Demographic information was obtained from patient records. There is some debate over the validity of pregnant smokers' reports of their smoking status [24] and a series of questions are sometimes used to increase smoking disclosure [25]. However, in this survey the intention was to confirm the smoking status recorded at the first antenatal visit and there was no reason to believe that the women would wish to deceive the interviewer when they had declared themselves as smokers at the antenatal visit. Therefore, all the women were asked, 'Can I just check, are you still smoking at the moment'? ('yes' or 'no'). Those confirming that they were still smoking were asked, 'About how many a day would you say you are smoking at the moment'?, and 'Are you thinking at all about stopping'? ('yes' or 'no'). Those expressing an interest in stopping were asked, 'Do you think you might want to stop in the next month, or might you prefer to try a bit later on' and 'Would you be interested in receiving some help from the hospital with stopping'? ('yes' or 'no'). Those smokers stating an interest in receiving help were asked, 'Would you be interested in any of the following types of help ('yes' or 'no' in each case): (a) a telephone help line, (b) self-help booklets, (c) individual counseling, support and advice, and (d) a stop smoking support group. Smokers reporting an interest in both individual and group support (c and d above) were asked, 'Would you prefer individual or group appointments, or don't you have a preference'?

Those reporting an interest in receiving help with stopping were also asked: (a) 'We are looking into personal exercise plans as a way of helping women in your position to stop smoking. Is this something you would be interested in'? (four point scale: 'not interested' (1), 'quite interested' (2), 'very interested' (3), 'extremely interested' (4)); and (b) 'We are also looking into whether having a pregnant 'buddy' who is also trying to stop smoking can help women in your position to stop. Is this something that you would be interested in'? ('not interested' (1) to 'extremely interested' (4)).

\subsection{Data analysis}

All data were analysed using SPSS version 10. Relationships between demographic characteristics, smoking characteristics and preferences for stop smoking support were examined using chi-squared tests $\left(\chi^{2}\right)$, analysis of variance (ANOVA) and Pearson's correlation coefficients. Multiple regression analyses was used to examine associations between variables controlling for other variables.

\section{Results}

The participant flow for the survey is presented in Fig. 1. The demographic characteristics of those women interviewed are given in Table 1.

\subsection{Interest in different types of smoking cessation support}

When asking the women about their interest in different types of stop smoking support (see Table 2), the 


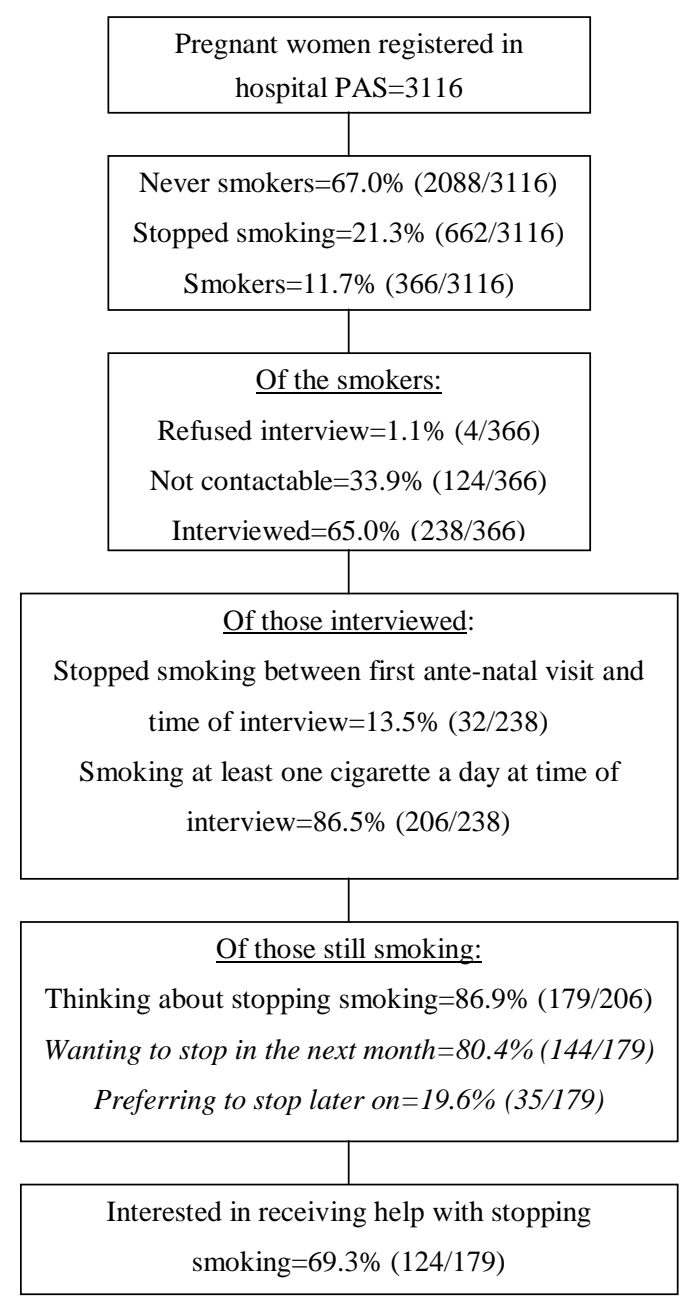

PAS=Patient Administration System

Fig. 1. Participant flow.

vast majority of women said that they were interested in face-to-face behavioural support and self-help booklets. Roughly half of the women reported being interested in using a telephone help-line. Around half of the women expressed a strong interest in having a personal exercise plan ('very' or 'extremely' interested in exercise plan $=46.7 \%$ (58/124); mean (S.D.) score for interest in exercise plan $=$

Table 1

Demographic characteristics of those women interviewed $(n=238)$

\begin{tabular}{ll}
\hline Characteristic & Mean (S.D.) \\
\hline Age (15-45 years) & $27.8(6.6)$ \\
Weeks of pregnancy (7-29 weeks) & $17.9(4.5)$ \\
Self-report of cigarettes smoked per & $8.3(5.5)$ \\
$\quad$ day (1-30 cigarettes) & \\
& Percentage (no.) \\
\hline Professional/managerial & $17.6(42)$ \\
Caucasian & $76.1(181)$ \\
First antenatal visit in hospital $\quad 71.0(169)$ \\
$\quad$ (rather than in a community clinic)
\end{tabular}

$2.4(0.1)$, range $=1-4)$. Approximately one-third of the women reported a strong interest in having a 'buddy' ('very' or 'extremely' interested in 'buddy' system $=35.7 \%$ (41/115); mean (S.D.) score for interest in 'buddy' $=2.1$ $(0.1)$, range $=1-4)$. A binomial test showed that significantly more women preferred individual as opposed to group appointments $(P=0.047)$.

\subsection{Relationship between demographic characteristics and interest in smoking cessation support}

The proportion of women expressing an interest in receiving help with stopping smoking (see Fig. 1) was significantly higher for those in professional/managerial occupations than in other occupations $\left(\chi^{2}=3.7, P=0.037\right.$, interested in help: professional $/$ managerial $=84.8 \%, 28 / 33$; non-professional $/$ managerial $=66.2 \%, 96 / 145)$, and was significantly higher for those who reported being heavier smokers $(F=5.3, P=0.022$; mean (S.D.) cigarettes per day: interested in help $(n=124)=8.7(5.6)$, not interested in help $(n=55)=6.8$ (3.9)). Using a forced entry multiple logistic regression analysis both occupation and smoking rate remained significantly associated with 'interest in receiving help' $(\mathrm{OR}=3.1, P=0.028$; $\mathrm{OR}=0.9$, $P=0.021$, respectively). There were no other significant associations between the demographic variables and smoking characteristics, interest in stopping smoking, or interest in receiving help with stopping.

Non-Caucasians reported significantly more interest in both individual counseling $\left(\chi^{2}=7.6, P=0.006\right.$; Caucasian $=65.2 \%, 60 / 92 ;$ non-Caucasian $=90.6 \%$,

Table 2

Reports of interest in different types of smoking cessation support

\begin{tabular}{llr}
\hline Variable & $\begin{array}{l}\text { Percentage } \\
\text { (no.) }\end{array}$ & $95 \%$ C.I.s \\
\hline Telephone help-line $(n=124)$ & $45.2(56)$ & $36.4-53.9$ \\
Self-help booklets $(n=124)$ & $76.6(95)$ & $69.2-84.1$ \\
Individual counseling, support \& advice & $71.8(89)$ & $63.9-79.7$ \\
$\quad(n=124)$ & & \\
A stop smoking support group $(n=124)$ & $62.9(78)$ & $54.4-71.4$ \\
Behavioural support $(n=124)$ & $82.3(102)$ & $75.5-89.0$ \\
$\quad$ group/individual support combined) & & \\
Prefers individual appointments $(n=102)$ & $40.2(41)$ & $30.7-49.7$ \\
Prefers group appointments $(n=102)$ & $22.6(23)$ & $14.4-30.7$ \\
No appointment preference $(n=102)$ & $37.2(38)$ & $27.9-46.6$ \\
Personal exercise plans $(n=124)$ & & \\
$\quad$ Not interested & $15.3(19)$ & $8.9-21.7$ \\
$\quad$ Quite interested & $37.9(47)$ & $29.4-46.4$ \\
$\quad$ Very interested & $29.0(36)$ & $21.0-37.0$ \\
$\quad$ Extremely interested & $17.7(22)$ & $11.0-24.5$ \\
Buddy'a system $(n=115)$ & & \\
$\quad$ Not interested & $30.4(35)$ & $22.0-38.8$ \\
Quite interested & $33.9(39)$ & $25.3-42.6$ \\
$\quad$ Very interested & $26.1(30)$ & $18.1-34.1$ \\
Extremely interested & $9.6(11)$ & $4.9-16.5$ \\
\hline
\end{tabular}

${ }^{\text {a }} n<124$ as this item was a late addition to the survey questionnaire. 
29/32) and group support $\left(\chi^{2}=11.2, P=0.001\right.$; Caucasian $=54.3 \%, 50 / 92 ;$ non-Caucasian $=87.5 \%$, 28/32). Those who were not in professional/managerial occupations were likely to report greater interest in 'buddying' $(F=4.2, P=0.042$; mean (S.D.) scores for interest in buddy: professional/managerial $(n=24)=1.8(0.9)$, non-professional/managerial $(n=91)=2.2(0.9))$. There were no other significant associations between the demographic characteristics and reports of interest in different types of smoking cessation support.

\section{Conclusion}

Overall, the results indicate that, consistent with previous findings for pregnant smokers [4], the large majority of women wanted to stop smoking in the next month. The vast majority of women expressed an interest in most types of smoking cessation support. Interest in this support was highest among heavier smokers and among those from professional/managerial occupations. Of the various types of smoking cessation support discussed, face-to-face behavioural support and self-help materials attracted the most interest. These results are consistent with previous findings that female smokers are likely to express a high level of interest in behavioural smoking cessation support, and are likely to express greater interest in this support than men [21]. In the present survey the level of interest reported for self-help material versus behavioural support was similar, a finding that is in contrast with a previous survey, of smokers in general, which found a strong preference for self-help materials over behavioural support [21]. Additionally, there was a preference for individual over group support, those from lower socio-economic groups reported more interest in 'buddying', and those from ethnic minorities reported the most interest in behavioural support.

\section{Discussion}

\subsection{Sample characteristics}

The study sample included a substantial proportion of ethnic minorities and women from more deprived socio-economic groups (see Table 1), and was similar to national samples of pregnant smokers drawn from across the UK [4]. Fewer women were recorded as smokers at their first antenatal booking visit than would be expected from national data [4]. This is likely to be due to underreporting of smoking [24] and also due to the high proportion of Asian women in the local population. Around 13\% of those registered as smokers at the booking visit reported stopping smoking by the time of the interview (see Fig. 1). This finding concurs with previous evidence showing that a substantial percentage of women are likely to spontaneously cease smoking during the early stages of pregnancy [4].
The average smoking rate for the study population of about eight cigarettes a day is similar to other recent studies where recruitment has followed the first antenatal visit [26,27].

\subsection{Interest in different types of smoking cessation support}

That heavier smokers expressed greater interest in receiving help with stopping smoking is consistent with evidence that heavier smokers are more likely to attend smoking cessation programmes [10]. This is promising since it is heavier smokers who are likely to find it most difficult to stop during their pregnancy $[28,29]$. That those in professional/managerial occupations reported most interest in receiving help with stopping may be related to these women perceiving fewer barriers (e.g. lack of child care) to attending clinics.

This survey reports a high level of interest among pregnant smokers in behavioural support for smoking cessation. Yet, according to previous studies, rates of uptake of this type of support are low [8-10]. Previous reports suggest that the reasons given for poor uptake of behavioural smoking cessation support among pregnant smokers include lack of social support and inadequate childcare [11]. Further work is needed to more comprehensively identify the barriers to uptake of smoking cessation interventions among pregnant smokers and to design interventions which address these barriers.

That more women were interested in individual than group appointments confirms previous reports $[3,30]$ and suggests that in order to maximise uptake rates pregnant smokers should be routinely offered individual appointments. Individual appointments offer flexibility, and provide privacy for pregnant women who may feel embarrassed about their smoking status.

Encouragingly, large numbers of the women were interested in self-help materials, an intervention which is relatively cheap and may have some effect in aiding smoking cessation [3]. Around half the women expressed a strong interest in an exercise intervention for smoking cessation. The offer of exercise support may be one way of attracting pregnant smokers to cessation programmes, as well as having the potential to enhance rates of smoking abstinence [13] and to improve general health during pregnancy [31]. Nearly a third of the smokers reported a strong interest in the 'buddy' system, suggesting that this intervention may have potential for pregnant smokers. Those from a lower socio-economic bracket, as indicated by occupation, were more interested in 'buddying'. This may reflect a situation where women from more deprived socio-economic groups experience a deficit of social support [11] and therefore report an increased desire for additional support.

\subsection{Study limitations}

This survey is limited in that reports of being interested in smoking cessation support are distinct from uptake of that 
support. The ultimate test of the acceptability of different smoking cessation interventions for pregnant smokers will be the degree to which different interventions are utilised. This study is also limited in that it relies on data from a single brief telephone interview. In order to corroborate the present findings it would be useful to repeat this study with more in-depth, and face-to-face, interviews.

At the outset, in the present study, no specific hypotheses were generated concerning the relationships between the demographic characteristics and other variables. Consequently, with so many statistical comparisons the chances of finding spurious relationships between these variables was reasonably high. Therefore, these findings require replication with larger samples sizes.

Finally, it is recognised that this survey is limited in that the women were given a restricted set of smoking cessation options to respond to, options which were largely limited to the type of support that might be considered for nationally funded smoking cessation services based in the UK. Further survey work needs to be carried out which targets other populations of pregnant smokers and which addresses other smoking cessation options. For example, anecdotal reports suggest that many of these women may be interested in home visits and in stopping smoking alongside other family members [9]. Moreover, the level of interest among pregnant women in cessation support delivered via the internet [32], or via interactive software [33], also needs exploring. In the UK, nicotine replacement therapy (NRT) is now considered for pregnant smokers who it is judged could not stop otherwise [34]. However, the level of interest in NRT among these women is not known. Finally, research is required to investigate the relationship between psychosocial variables, such as self-efficacy [28] and social support for stopping smoking [35], and preferences for smoking cessation support among pregnant smokers.

\subsection{Practice implications}

The current survey is useful in that it gauges the level of interest in different types of stop smoking support for pregnant smokers, and therefore provides support for the utility of these interventions. The results reported here suggest that pregnant smokers may be attracted towards a range of smoking cessation interventions; namely, behavioural support, self-help materials, telephone help-lines, exercise, and 'buddy' support. Moreover, the findings suggest that 'buddy' support may be of particular interest to those in more deprived social groups and that ethnic minorities may be particularly attracted by behavioural support. In addition, uptake of smoking cessation support during pregnancy may be aided though routinely offering individual, as well as group, appointments for smoking cessation treatments.

Through creating interventions which are more acceptable to pregnant smokers these results may aid in increasing the uptake and effectiveness of smoking cessation support
[20]. For smokers in general, there is some evidence for the benefits of tailoring smoking cessation support to smokers' characteristics [36], although no studies could be identified which have tailored this support towards smokers' preferences for different types of support. Further studies are needed to examine the effectiveness of tailoring smoking cessation interventions towards different characteristics of pregnant smokers and towards these womens' preferences for different interventions.

\section{References}

[1] Castles A, Adams EK, Melvin CL, Kelsch C, Boulton ML. Effects of smoking during pregnancy: five meta-analyses. Am J Prev Med 1999;16:208-15.

[2] Adams EK, Melvin CL. Costs of maternal conditions attributable to smoking during pregnancy. Am J Prev Med 1998;15:212-9.

[3] Lumley J, Oliver S, Waters E. Interventions for promoting smoking cessation during pregnancy (Cochrane Review). In: The Cochrane Library, Issue 2. Oxford: Update Software; 2000.

[4] Owen LA, Penn GL. Smoking and pregnancy: a survey of knowledge, attitudes and behaviour, 1992-1999. London: Health Education Authority; 1999.

[5] US Department of Health and Human Services. The Health Benefits of smoking cessation. A report of the surgeon general. Rockville, Maryland: Public Health Service on smoking and health (DHHS Publication No. CCDC 90-8416); 1990.

[6] Haslam C, Draper ES, Goyder E. The pregnant smoker: a preliminary investigation of the social and psychological influences. J Public Health Med 1997;19:187-92.

[7] Silagy C. Physician advice for smoking cessation. In: The Cochrane Library, Issue 2. Oxford: Update Software; 2000.

[8] Hughes JR, Epstein LH, Andrasik F, Neff DF, Thompson DS. Smoking and carbon monoxide levels during pregnancy. Addict Behav 1982;7:271-6.

[9] Taylor T, Hajek P. Smoking cessation services for pregnant women. London: Health Development Agency; 2001.

[10] Lichenstein E, Hollis J. Patient referral to a smoking cessation program: who follows through? J Fam Pract 1992;34:739-44.

[11] Klerman LV, Spivey C, Raykovitch. Smoking reduction activities in a federal program to reduce infant mortality among high-risk women. Tob Control 2000;9(Suppl III):51-5.

[12] Zhu SH, Stretch V, Balabanis M, Rosbrook B, Sadler G, Pierce JP. Telephone counseling for smoking cessation: effects of singlesession and multiple-session interventions. J Consult Clin Psychol 1996;64:202-11.

[13] Ussher MH, West R, Taylor AH, McEwen A. Exercise interventions in smoking cessation. In: The Cochrane Library, Issue 3. Oxford: Update Software; 2000.

[14] May S, West R. Do social support interventions ("buddy systems") aid smoking cessation? A review. Tob Control 2000;9:415-22.

[15] Albrecht S, Payne L, Stone CA, Reynolds MD. A preliminary study of the use of peer support in smoking cessation programs for pregnant adolescents. J Am Acad Nurse Pract 1998;10:119-24.

[16] West R. Smoking cessation and pregnancy. Fetal Matern Med Rev 2002;13:184-94.

[17] Walsh RA, Redman S. Smoking cessation in pregnancy: do effective programs exist? Health Promot Int 1993;8:111-26.

[18] Lefebvre R, Flora J. Social marketing and public health intervention. Health Educ Quart 1988;15:299-315.

[19] Strechter V, Rimer B, Monaco K. Development of a new self-help guide: freedom from smoking for you and your family. Health Educ Quart 1989;16:101-12. 
[20] Floyd RL, Rimmer BK, Giovino GA, Mullen PD, Sullivan SE. A review of smoking in pregnancy: effects on pregnancy outcomes and cessation efforts. Ann Rev Pub Health 1993;14:379411.

[21] Owen N, Davies MJ. Smokers' preferences for assistance with cessation. Prev Med 1990;19:424-31.

[22] Spoth R. Formative research on smoking cessation program attributes preferred by smokers. Am J Health Promot 1991;5:346-54.

[23] Spoth R. Smoking cessation program preferences associated with stage of quitting. Addict Behav 1991;16:427-40.

[24] Campbell E, Sanson-Fisher R, Walsh R. Smoking status in pregnant women assessment of self-report against carbon monoxide (CO). Addict Behav 2001;26:1-9.

[25] Mullen PD, Carbonari JP, Tabak ER, Glenday MC. Improving disclosure of smoking by pregnant women. Am J Obstet Gynecol 1991;165:409-13.

[26] Ershoff DH, Solomon LJ, Dolan Mullen P. Predictors of intentions to stop smoking early in pre-natal care. Tob Control 2000;9(Suppl III):41-5.

[27] Hajek P, West R, Lee A, Foulds J, Owen L, Eiser JR, et al. Randomized controlled trial of a midwife-delivered brief smoking cessation intervention in pregnancy. Addiction 2001;96:48594.
[28] Woodby LL, Windsor RA, Snyder SW, Kohler CL, DiClemente CC. Predictors of smoking cessation during pregnancy. Addiction 1999;94:283-92.

[29] Lu Y, Tong S, Oldenburg B. Determinants of smoking and cessation during and after pregnancy. Health Promot Int 2001;16:355-65.

[30] O'Connor AM, Davies BL, Dulberg CS, Buhler PL, Nadon C, McBride $\mathrm{BH}$, et al. Effectiveness of a pregnancy smoking cessation program. J Obstet Gynecol Neonatal Nurs 1992;2:385-92.

[31] Artal R, Sherman C. Exercise during pregnancy: safe and beneficial for most. Phys Sports Med 1999;27:51-60.

[32] Etter JF. Using new information technology to treat tobacco dependence. Respiration 2002;69:111-4.

[33] Scott WJ, McIlvain H. Interactive software: an educational/behavioural approach to smoking cessation for pregnant women and their families. Tob Control 2000;9(Suppl 3):56-7.

[34] West R, McNeill A, Raw M. Smoking cessation guidelines for health professionals: an update. Thorax 2000;55:987-99.

[35] Dejin-Karlsson E, Hanson BS, Ostergren PO, Ranstam J, Isacsson SO, Sjoberg NO. Psychosocial resources and persistent smoking in early pregnancy: a population study of women in their first pregnancy in Sweden. J Epidemiol Commun Health 1996;50:33-9.

[36] Lancaster T, Stead LF. Self-help interventions for smoking cessation. In: The Cochrane Library, Issue 3. Oxford: Update Software; 2002. 